\title{
ON AN AXIOM OF BOURBAKI
}

\author{
OSWALD WYLER
}

It is the purpose of this paper to show that Axiom A2 in Bourbaki's axiomatic system for set theory can be replaced by the weaker statement that every term $x$ defines a set $\{x\}$ of which $x$ is the only element. All references in the paper are to [1], and the terminology and notation of [1] are used.

We place ourselves into a theory with specific signs $=$ and $\epsilon$, in which S1 through S8 are schemas, and A1, A4 and the statement

$$
(\forall x) \operatorname{Coll}_{y}(y=x)
$$

are axioms. The theory may contain other specific signs, schemas and axioms.

Axiom (1) allows us to define a set $\{x\}=E_{y}(y=x)$. Criterion C51 (p. 65) then can be proved. It follows that there is a set $\phi$ such that $(\forall x)(x \notin \phi)$ is true. Sets $\{\phi\}$ and, using A4, $\mathfrak{P}(\{\phi\})$ can then be constructed. It follows that $\phi \in \mathfrak{P}(\{\phi\}),\{\phi\} \in \mathfrak{P}(\{\phi\})$, and $\phi \neq\{\phi\}$.

Let now $R$ in S8 (p. 64) be the relation

$$
(x=u \text { and } y=\phi) \text { or }(x=v \text { and } y=\{\phi\}) \text {. }
$$

The relation

$$
(\forall y)(\exists X)(\forall x)(R \Rightarrow(x \in X))
$$

is true, as we may put $X=\{u\}$ for $y=\phi$ and $X=\{v\}$ for $y \neq \phi$. Then

$$
\operatorname{Coll}_{x}((\exists y)((y \in \mathfrak{B}(\{\phi\})) \text { and } R))
$$

is true, by $\mathrm{S} 8$ and C30 (p. 37). Since

$$
((\exists y)((y \in \mathfrak{P}(\{\phi\})) \text { and } R)) \Leftrightarrow(x=u \text { or } x=v) \text {, }
$$

we have $\operatorname{Coll}_{x}(x=u$ or $x=v$ ), and hence, using C27 (p. 36),

$$
(\forall u)(\forall v) \operatorname{Coll}_{x}(x=u \text { or } x=v) .
$$

This is a restatement of A2.

\section{REFERENCE}

1. N. Bourbaki, Théorie des ensembles, Chap. I and II., Actualités Scientifiques et Industrielles, no. 1212, Paris, Hermann, 1954.

University of New Mexico

Received by the editors October 5, 1956. 